$\begin{array}{cl}\begin{array}{c}\text { Revue } \\ \text { de /histoire }\end{array} & \text { Revue de l'histoire des religions } \\ \text { des religions } & \begin{array}{l}3 \mid 2015 \\ \text { Varia }\end{array}\end{array}$

\title{
La biographie spirituelle : une littérature de figuration
}

Notes critiques

Spiritual Biography: a figurative literature

\section{Antoinette Gimaret}

\section{OpenEdition}

\section{Journals}

Édition électronique

URL : http://journals.openedition.org/rhr/8411

DOI : $10.4000 /$ rhr.8411

ISSN : 2105-2573

\section{Éditeur}

Armand Colin

\section{Édition imprimée}

Date de publication : 1 septembre 2015

Pagination : 363-371

ISBN : 978-2-200-92993-0

ISSN : 0035-1423

\section{Référence électronique}

Antoinette Gimaret, « La biographie spirituelle : une littérature de figuration », Revue de l'histoire des religions [En ligne], 3 | 2015, mis en ligne le 01 septembre 2018, consulté le 19 avril 2019. URL : http:// journals.openedition.org/rhr/8411; DOI : 10.4000/rhr.8411 


\section{La biographie spirituelle: une littérature de figuration Notes critiques"}

Jacques Le Brun, spécialiste des biographies spirituelles féminines du XVII siècle français, a réuni en un volume une dizaine d'articles consacrés à ce corpus spécifique. Il en éclaire les principaux enjeux à la fois spirituels, littéraires et anthropologiques et montre dans quelle mesure le genre biographique peut se définir comme une entreprise de (re)figuration de l'expérience spirituelle, une expérience qui se situerait elle-même toujours au carrefour d'une appartenance confessionnelle, d'une érotique et d'un fait de discours. Outre sa grande richesse méthodologique, ce recueil d'articles offre une réflexion passionnante sur l'usage des "lieux communs » dans le champ de la spiritualité et sur la place centrale dévolue au corps féminin, à la fois creuset et écueil de la sainteté.

\section{Spiritual Biography: a figurative literature}

Jacques Le Brun, a specialist in feminine spiritual Biographies of the French XVIIth Century, gathered in one volume a dozen articles dedicated to this specific corpus. He sets out its main issues at once spiritual, literary and anthropological and demonstrates how the biographical genre can define itself as a (re)figuration of spiritual experience, at the crossroads of religious affiliation, erotic and fact of speech. Besides its methodological wealth, this volume offers a fascinating reflection on the use of "topoi" in spirituality and the role assigned to the female body.

* À propos de Jacques Le Brun, Sæeur et amante. Les biographies spirituelles féminines du XVII siècle, Genève, Droz, 2013, 273 p., ISBN 978-2-600-00552-4. 
La biographie spirituelle est un genre abondant dans toute l'Europe catholique du XVII' siècle. En effet, si le Concile de Trente a conduit au renouveau d'une hagiographie officielle centrée sur les saints fondateurs et les grandes figures de la Contre-Réforme, il a favorisé également la rédaction, à l'intérieur des couvents féminins, de milliers de Vies restées le plus souvent manuscrites et consacrées à des figures de «spirituelles» majoritairement en marge de la sainteté reconnue. À la fois documents historiques, témoignages hagiographiques et textes littéraires, ces biographies sont essentielles pour comprendre les représentations et les pratiques spirituelles de cette époque. Jacques Le Brun, qui en explore depuis plus de trente ans le versant français, a réuni en un volume une dizaine d'articles consacrés à ce genre complexe $e^{1}$. On ne peut que saluer cette mise en recueil, qui souligne la cohérence du parcours de recherche de l'auteur et confirme une approche fondatrice de ces textes.

\section{Des PISTES MÉTHOdologiQues}

Il faut tout d'abord insister sur la richesse méthodologique de l'ouvrage. En même temps qu'il les analyse, l'auteur propose une réflexion sur les voies d'approche possibles de ces Vies. Les méthodes statistiques se trouvent insuffisantes voire inefficaces, souligne-t-il, pour un corpus aux limites toujours floues, de l'ordre le plus souvent de l'archive manuscrite et aux processus d'édition complexes. Jacques Le Brun montre la nécessité d'en multiplier les approches en usant des outils de la sociologie, de l'historiographie, de la psychanalyse, de la théologie ou encore de la médecine. Seule la convocation croisée de ces savoirs permettrait de saisir quelque chose d'un «contenu» biographique, d'une expérience spirituelle qui se situerait au carrefour de l'individuel et du collectif, de l'institutionnel et de l'intime. Les biographies spirituelles, liées au

1. L'ouvrage se compose de onze chapitres (neuf textes remaniés, deux textes inédits) précédés d'une introduction générale exposant les enjeux du genre biographique. 
développement des congrégations et ordres féminins, permettraient d'appréhender en effet des «formes particulières de vie intérieure», une «façon de vivre le christianisme propre à ces sociétés féminines tout en reflétant les grandes tendances de la spiritualité du temps » (p. 7). Elles manifesteraient donc tout autant une singularité (des pratiques, d'un itinéraire de vie) qu'un ancrage institutionnel, générique ou social. Le récit de vie comme «geste de fondation $»^{2}$ ferait le lien entre le «corps institué» qu'est l'ordre religieux et le corps singulier de la religieuse, elle-même vivante image de la sainte fondatrice qu'elle prend pour modèle. C'est au carrefour du corps, du texte et de l'institution que la spiritualité comme expérience peut s'écrire. Mais c'est bien «comme littérature que nous devons considérer ces textes» rappelle Jacques Le Brun (p. 204). La biographie est à appréhender comme un «genre littéraire» (p. 45) et l'expérience spirituelle avant tout comme un «fait de discours », car la seule réalité aujourd'hui pour nous est «ce texte» (p. 13). L'auteur met donc en garde contre cette erreur qui guette le chercheur: croire saisir, dans ce qui se donne comme écriture du témoignage, un fait brut. "Ce que nous interprétons en une lecture inattentive comme expériences individuelles apparaît à l'analyse comme traitement de thèmes littéraires, la seule expérience alors perceptible est celle de l'écriture, celle du biographe introduisant un ordre théologique, dramatique ou esthétique dans le matériau documentaire dont il disposait» (p. 45), travail de réécriture qui déporte la biographie de l'historiographie vers la littérature.

Par là l'ouvrage construit véritablement son objet et met en lumière la richesse du genre biographique, dans la mise en avant du travail de figuration que suppose le récit de vie: processus de la transformation du témoignage en aveu au chapitre III ( «L'aveu», p. 61-76), processus d'autorisation de la sainteté au chapitre IX («La sainteté à l'époque classique et le problème de l'autorisation», p. 241-260), inscription de la Vie sainte dans une dynamique rétrospective. Le chapitre II («Conversion et continuité intérieure», p. 43-59) montre par exemple comment le schéma de continuité l'emporte le plus souvent dans ces textes sur le schéma de rupture qu'implique la conversion, le biographe usant des topoi de la prédestination ou de l'hérédité

2. Louis Marin, «Biographie et fondation», dans Pascal et Port Royal, Paris, PUF, 1997, p. 387-402, cité p. 10. 
sainte afin de reconstruire «un itinéraire dont l'unité est assurée par des discours qui derrière le temps des événements suppose un temps fixe», temps qui n'est plus celui de la chronologie historique (p. 48). Jacques Le Brun insiste également, dans ce travail de figuration, sur l'importance du regard distancié du biographe qui rend lisible l'expérience corporelle puisque «seule l'autre peut dire le corps de l'autre» (p. 116). Trouvant son origine dans le regard fasciné d'un témoin qui ne peut d'abord que «fixer sans comprendre» (p. 28), le texte biographique s'écrit toujours dans l'après-coup, comme (ré)élaboration, (re)figuration de l'expérience spirituelle. Ainsi les altérations du corps, interprétées comme traduction visible des états intérieurs, ne sauraient-elles trouver un sens dans l'immédiateté de leur perception. En effet, la manifestation corporelle visible pour autrui est en elle-même «dépourvue d'un discours interprétatif» (p. 71); ce n'est qu'a posteriori, dans le discours qui en rend compte, dans le «lien établi entre les confidences faites à autrui et la description des altérations du corps» que le sens s'élabore, «la biographie [cherchant et élaborant] un discours [...] pour le visible» (p. 73). Seul ce travail de figuration permet à la souffrance de devenir «martyre» car «un autre reçoit ce témoignage de souffrance et $[. .$.$] lui donne sens» (p. 160). Jacques Le Brun éclaire dans$ cette perspective l'importance de logiques métaphoriques servant à suggérer des causalités mystiques et à ériger le corps pathologique en Imitatio Christi ${ }^{3}$.

Alerté sur le rôle essentiel du biographe dans l'élaboration du sens, le lecteur d' aujourd' hui est alors en mesure de dépasser le débat «récurrent mais futile sur la "réalité" des phénomènes mystiques» (p. 103) afin de comprendre comment s'élabore l'expérience spirituelle comme discours, autrement dit quel lien s'établit entre le texte et un savoir - doctrinal, médical, historique - qui lui préexiste et non pas entre le texte et un réel dont il devrait rendre compte ${ }^{4}$. Seul le repérage de ce travail de figuration lui permettra de saisir, entre les lignes de la matière biographique, les contours d'une expérience qui est surtout élaboration et mutation d'un discours ${ }^{5}$.

3. Voir p. 196-197.

4. Voir p. 23: «Dire un "rapport" entre un écrit et un savoir, non pas entre un compte rendu et une réalité antérieure».

5. Voir p. 24: «Ce qui se dit dans les biographies c'est moins un contenu [...] que l'acte de dire [...], ce que ces femmes appellent une "expérience"». 


\section{Du CAS INDIVIDUEL À LA FIGURE : RÉFLEXIONS SUR LE LIEU COMMUN}

Passionnante dans ce contexte est la réflexion que propose Jacques Le Brun sur le lieu commun en tant que méthode d'intégration de la singularité dans une narration qui serait le récit de vie. Le corpus biographique étant «l'articulation [...] de l'individuel et du collectif, de l'individuel et de l'institué» (p. 13), il faut à la fois faire du «cas par cas» et trouver "l'unité dans une série», c'est-à-dire trouver un langage critique capable d'articuler le «singulier du fait avec la durée» (p. 15). En effet, «même unique [...] chaque vie prend place dans la longue histoire de l'écriture des vies». Il s'agira donc d'inscrire ces Vies singulières dans une tradition (par le repérage de «lieux communs hagiographiques», exempla, ou stéréotypes), tout en mettant en lumière les réaménagements, déplacements ou réécritures de ces mêmes topoi. Par ce maniement du lieu commun, les biographies apprendront «de ces vies ce qu'elles ont en commun avec les autres», dans la «continuelle réduplication de modèles canoniques». En même temps, elles diront quelque chose d'une rupture possible, d'un réaménagement des représentations dans le champ de la spiritualité au XVII ${ }^{\mathrm{e}}$ siècle.

Différents motifs (le martyre, la sainteté, la conversion ou encore la stigmatisation) sont ainsi travaillés par l'auteur non en tant que réalités mais en tant que «topoi hagiographiques » à l'intérieur d'un genre canonique reposant lui-même sur une logique mémorielle et imitative ${ }^{6}$. Jacques Le Brun parvient ainsi à poser la question d'une «modernité» qui serait à l'œuvre dans un champ pourtant marqué par des schémas de continuité et de fidélité à la tradition. On soulignera dans ce contexte la richesse de certaines analyses comme celle des mutations du martyre («Mutations de la notion de martyre au XVII ${ }^{e}$ siècle d'après les biographies spirituelles », p. 157175) ou encore de l'évolution du topos du baiser au lépreux devenu, dans sa variante «moderne», baiser au chancreux ${ }^{7}$ : l'expérience spirituelle trouverait à se dire dans ces nouveaux discours intégrant une forme d'actualité, à la jonction de la singularité et du stéréotype, du topique et de l'inédit.

6. Voir p. 12: «Les religieuses sont ou doivent être la réincarnation des fondateurs et, au-delà, de toutes les saintes dont elles portent les noms, ultimement de Jésus-Christ».

7. Voir p. 189-190. 
Trait de modernité évoqué de façon récurrente, la pathologisation du principe d'Imitatio Christi, discours théologique et médical se conjuguant afin de retravailler le topos du corps souffrant: les maladies comme participation à la Passion du Christ peuvent se substituer ici aux pratiques ascétiques et aux phénomènes corporels extraordinaires, ainsi des lésions cancéreuses au sein assimilées à la plaie stigmatique au côté. Si les biographies spirituelles reproduisent une logique imitative commune, elles opèrent une «mutation du topos hagiographique» en faisant ainsi du cancer une «nouvelle maladie canonique» (p. 200). De même le martyre ne disparaît pas dans sa structure mais devient une «mise en scène du corps souffrant organisé par la médecine et la chirurgie» (p. 128) dans un contexte tridentin où l'aspiration au martyre ne saurait plus se concrétiser dans sa forme ancienne. Il reste présent, mais au prix de déplacements qui instaurent de nouveaux lieux communs de la spiritualité, comme le martyre par mortification (p. 158-161) ou le martyre chirurgical (p. 161-169). Un autre exemple parlant de ces mutations est celui de la stigmatisation. Le chapitre VI (p. 131-155) est entièrement consacré à l'évolution de cette notion, évolution qui n'est pas seulement à interpréter comme une marque de prudence doctrinale mais bien comme l'indice d'un changement de mentalités à l'intérieur même du discours religieux, dans la cristallisation progressive d'un autre lieu commun, celui des «stigmates intérieurs et invisibles», témoignant d'une «mutation à la fois épistémologique et spirituelle» puisque là où la visibilité était le critère même de l'existence du phénomène prennent place d'autres critères, ceux de la faiblesse et de la souffrance du corps ${ }^{8}$.

\section{MUtATIONS ET RÉVERSIBILITÉS DU SIGNE CORPOREL}

Le révélateur essentiel de ces changements de mentalités, de ces déplacements de stéréotypes est essentiellement ici le corps, dont les biographies dévoilent à la fois la richesse signifiante et les ambiguïtés. Dans son chapitre V («À corps perdu» p. 105-130), l' auteur souligne que la production du discours biographique s'origine toujours dans une perte première, la Vie ne pouvant s'écrire qu'à partir de la mort

8. Voir l'étude du cas célèbre de Marie de l'Incarnation, p. 147-148. 
de la religieuse, elle-même dans le souvenir d'un Dieu incarné dont il faut faire le deuil dans le réel. Elle se justifie donc comme une entreprise de reconstruction de corps, de création de présence. S'il s'agit de «combler l'horreur de la perte» (p. 105) par l'Eucharistie ou encore par l'usage des images, les religieuses font également de leur propre corps le lieu de surgissement de cette Présence. Alors que le Christ paraît «de moins en moins vivant dans le monde et la société» (p. 123), elles se vouent à le «faire surgir dans le réel», à le rendre présent en elles, dans une imitation littérale étonnante (l'auteur insistant à de nombreuses reprises sur l'engagement de «prendre à la lettre» le texte biblique ${ }^{9}$ ). On est bien à nouveau ici dans une entreprise de figuration: parce que le désir d'Imitation du Christ «se cherche une forme» (p. 127), le corps devient figure car «support d'une ressemblance $»^{10}$.

Mais le corpus des Vies illustre également la réversibilité du signe corporel, la part d'incontrôlable qu'il recèle (dérive vers une érotique, perte de sens dans l'excès d'horreur ou l'excès pathologique) et la nécessité de gérer cet extraordinaire afin qu'il reste acceptable et n'invalide pas le processus d'autorisation de sainteté. Le chapitre IX («Horreur et jouissance», p. 203214) illustre bien ce passage à l'excès, lorsque la mortification s'éloigne des pratiques contrôlées pour verser dans une «horreur systématique» (p. 205) recherchée par la religieuse de sa propre initiative et dont Jacques Le Brun donne quelques exemples parlants: autoflagellation, consommation d'ordures et de crachats, nettoyage de plaies purulentes avec la langue. Si cette horreur peut être dépassée dans la pénitence et la perspective de récompenses célestes, l'auteur souligne que ces compensations sont loin d'être toujours évoquées ${ }^{11}$, la biographie devenant alors cette parole d'excès, de désir inavouable de l'horreur qui trouve sa réalisation dans l'imitation littérale des exempla les plus extraordinaires. Difficile de rendre acceptable ce comportement ascétique extrême, parfois décroché de toute interprétation spirituelle et basculant

9. Voir par exemple p. 123 à propos du nom du Christ gravé au canif ou marqué au fer rouge sur la chair.

10. Voir p. 126-127 à propos du corps pathologique: «Loin de rendre caduque cette identification, la mutation médicale permet la dissémination des plaies et des souffrances, composant des lieux ou peut s'inscrire l'image».

11. Voir p. 208. 
peu à peu dans le pathologique, comme le suggère par exemple le cas de Louise du Néant, internée à la Salpêtrière. Il relèverait en effet davantage d'une «pulsion d'autodestruction» (p. 210) dont l'horreur ne serait compensée qu'après coup par le recours à l'image de la Croix ou des noces célestes.

L'auteur souligne alors la nécessité pour les biographes de recourir à des procédures d'acceptabilité, en premier lieu l'introduction de la notion de charité pratique, qui rend utile l'horreur et fait basculer l'excès ascétique en geste thérapeutique (par exemple le baiser au malade qui devient un remède nettoyant la plaie, p. 213), dans une «naturalisation» des gestes de l'horrible. Autre domestication possible de l'extraordinaire corporel, l'équilibre gardé entre dévoilement et secret, ainsi de cette invention des stigmates invisibles qui permet le passage de la plaie sanglante à la douleur comme effet de discours. Le corps continue ici de manifester une présence mais dans la similitude, le comme si, manifestant donc à la fois la présence et la «radicale impossibilité de la présence $»^{12}$. Le corps visible fait signe vers un ailleurs qui le déborde et dont même l'interprétation pathologique ne peut plus exténuer le sens. Les biographies spirituelles invitent ainsi à dépasser l'évidence du spectacle corporel pour regarder un invisible, paradoxe qui fait la richesse de textes posant la lacune et la perte (de soi, du texte, du sens) comme fondatrices. C'est précisément par «cette façon de ruiner la totalité» (p. 31) que de tels textes offriraient les marques d'une écriture féminine ${ }^{13}$ : restant «en deçà de la totalité et du sens » (p. 30), cette écriture permettrait à une expérience de se dire, mais dans un «paraître» toujours problématique, dans une oscillation constante entre certitude et incertitude ruinant «toute prétention au tout ${ }^{14}$. La « ruine du tout» serait suggérée également par la logique de «sainteté insulaire» (p. 169) ou par la disparition des stigmates comme «signature d'ensemble» au profit d'une fragmentation, d'un «corps distribué en des lieux, en des organes qui deviennent chacun le support de signes» (p. 125).

12. P. 155. Sur cette notion de stigmates invisibles, voir notre étude «Corps marqué et stigmates invisibles dans les biographies spirituelles du XVII ${ }^{\mathrm{e}}$ siècle», Archivio italiano per la storia della pietà, XXVI, 2013, p. 239-258.

13. Voir le chapitre I «Écriture féminine? Écriture mystique?», p. 21-41.

14. P. 32. Voir aussi p. 31 : «Ce que nous découvrons dans ces vies c'est qu'il y a toujours un reste à la totalisation exigée par la théologie et l'institution». 
Cette suggestion d'un «reste» excédant la totalité visible ferait la richesse et la complexité du genre biographique par rapport à l'hagiographie officielle: genre littéraire obéissant à la fois à des logiques de sédimentation - où l'ancien ne cesse de se réactiver dans le nouveau et le lieu commun dans le singulier - et à des logiques de mutation par lesquelles certains phénomènes cessent peu à peu d'être acceptables, il dévoile de ce fait qu'il y a du «jeu» ou du «trop» dans les représentations, ainsi de ce trop-plein de souffrance inutilisable même dans l'hagiographie puisqu' aucun dogme «ne peut s'en renforcer, aucune vérité, seulement le réel de la souffrance». La biographie peut ainsi témoigner de la «coexistence de plusieurs visions du monde» (p. 214) à partir des mêmes lieux communs, ainsi du baiser au malade interprété à la fois comme extrême de l'horreur et comme extrême de la charité, coïncidence paradoxale témoignant très justement d'une logique spirituelle rétive à toute vision linéaire. Prenant acte de cette difficulté à repérer, au cours du $\mathrm{XVII}^{\mathrm{e}}$ siècle, des moments précis de bascule (du discours mystique au discours médical, du corps sanglant au corps intérieur, de l'imitation littérale à la métaphore), Jacques Le Brun choisit plutôt d'éclairer la perméabilité de ces textes aux autres discours, en particulier ceux de la médecine et met en lumière, contre tout schématisme hâtif, l'enchevêtrement des causalités, la convergence possible des discours ${ }^{15}$ ou encore la «réversibilité de l'autorité du topos hagiographique» qui peut aisément basculer en «contre-autorité» (p. 252-254), ainsi du signe corporel qui autorise la sainteté mais peut tout autant susciter le doute. Cette écriture troublée permettrait in fine de dire quelque chose d'un «fond obscur» de l'individu, de révéler «une vérité cachée qui n'a pas de place dans la confession sacramentelle» (p. 71-72), ainsi de la place du rêve comme saisie d'un inconscient (p. 102), de la pulsion de mort ou de la convocation de la catégorie de l'horreur qui, rappelle l'auteur, n'est pas théologique. C'est également cette part d'inavouable que l'ensemble de l'ouvrage parvient très efficacement à suggérer.

antoinette.gimaret@unilim.fr

15. Voir p. 164 à propos de la mutation chirurgicale du martyre (l'autorité discriminante n'est plus l'Église mais le médecin témoignant de l'héroïcité de la religieuse) et p. 248 (le discours médical sert de caution à l'idée de miracle). 